\title{
Review of: "Chromatin-based, in cis and in trans regulatory rewiring underpins distinct oncogenic transcriptomes in multiple myeloma"
}

Jean-Baptiste Alberge

Potential competing interests: The author(s) declared that no potential competing interests exist.

The authors share an analysis of chromatin accessibility and paired gene expression profiles of plasma cells from multiple myeloma patients and control individuals.

This is clearly an important field of research that still has too few published results. Accessibility to this work, together with that of others, is very much appreciated. The analytical methods are solid, and the organization of the manuscript is very clear.

Unfortunately, there is too little discussion of previous work in chromatin, epigenetics, and transcription (oncogenesis of $\mathrm{t}(4 ; 14)$ from the group of Florida, DNA methylation of B-cell enhancers from the Spanish group, molecular subgroups from UAMS and HOVON). Instead, the authors provide a very detailed description of their own analysis of the landscape of chromatin and gene expression.

Overall, it is a remarkable effort and good contribution to the field; it will certainly help researchers better understand myeloma oncogenesis and chromatin dysregulation in cancer.

Additional remarks:

-The list of genes most over-expressed in ND vs MM contains megakaryocyte, monocyte, and neutrophile markers and should be interpreted carefully. Similarly, only one locus is significantly more accessible in ND than in MM (but 7000 MM vs ND).

-Transcription factors and trans-acting factors seem to be used as equivalents by the authors? This is a questionable concept. Same for (B cell) differentiation versus developmental processes?

-Gene/locus-level details of LF loadings would be appreciated

-The conceptual differentiation between "de novo" and potentially "re-commissioned" enhancers throughout differentiation to malignant plasma cells is fascinating and should (hopefully) be described and discussed in a following paper! 